\title{
The Physical Conditions of the X-ray Emission Line Regions in the Circinus Galaxy
}

\author{
Masao Sako ${ }^{1}$, Steven M. Kahn ${ }^{1}$, Frits Paerels ${ }^{1,2}$, and Duane A. Liedahl ${ }^{3}$
}

\begin{abstract}
We present a detailed X-ray spectral analysis of the Circinus Galaxy using archival data obtained with the $A S C A$ satellite. The spectrum shows numerous emission lines in the soft X-ray band from highly ionized ions, as well as Compton reflection and fluorescent lines from neutral or near-neutral matter. We analyze the spectrum in the context of a self-consistent recombination cascade model and find that a nearly flat differential emission measure (DEM) distribution in ionization parameter fits the data. For a fixed solid angle distribution of matter surrounding a point source, this corresponds to a run of electron density of the form, $n(r) \sim r^{-3 / 2}$, which is suggestive of Bondi accretion onto a central compact mass. Using this density profile and comparing the resulting emission spectra with the $A S C A$ data, the size of the X-ray emission line region is estimated to be $\lesssim 1 \mathrm{kpc}$. We also show that the derived density as a function of radius is compatible with the X-ray recombination line emission region being the confining medium of the coronal line regions.
\end{abstract}

Subject headings: galaxies: individual (Circinus Galaxy) — galaxies: active — galaxies: Seyfert — galaxies: starburst — X-rays: galaxies

\section{Introduction}

X-ray spectra of Seyfert 2 galaxies show numerous discrete spectral features that are produced in a wide variety of physical conditions. In the few cases where the statistical quality of the data has permitted a detailed investigation of the spectrum, the soft X-ray band has been found to be almost completely dominated by line emission while the hard X-ray emission has been found to be consistent with nonthermal radiation possibly produced through accretion onto the central

\footnotetext{
${ }^{1}$ Columbia Astrophysics Laboratory and Department of Physics, Columbia University, 538 West 120th Street, New York, NY 10027; masao@astro.columbia.edu (MS), skahn@astro.columbia.edu (SMK), frits@astro.columbia.edu $(\mathrm{FP})$

${ }^{2}$ Laboratory for Space Research, Stichting Ruimte Onderzoek Nederland, Sorbonnelaan 2, 3584CA, Utrecht, Netherlands

${ }^{3}$ Department of Physics and Space Technology, Lawrence Livermore National Laboratory, P.O. Box 808, L-41, Livermore, CA 94550; duane@leo.llnl.gov
} 
compact object. X-ray observations have placed meaningful constraints on the structure of matter immediately surrouding the central source, and have played an important role in the development of a unified model of AGN (Antonucci 1993). Observations of warm absorbers in Seyfert 1 galaxies, for example, and their response to variations in the X-ray continuum provide compelling evidence that a significant amount of circumnuclear material is photoionized by the primary radiation (Otani et al. 1996). There is also observational evidence that a large fraction of Seyfert 2 galaxies harbor starburst regions where large amounts of mechanically-heated gas are produced, for example, through supernova explosions. Nuclear jets may also heat the surrounding gas through shocks. The orgin of the observed soft X-ray emission and the excitation mechanisms responsible for producing the observed emission lines in these galaxies, however, are not well understood and, in most cases, cannot be discerned from the low spectral resolution data available to date.

In the archetypal Seyfert 2 galaxy NGC 1068, for example, Ueno et al. (1994) find that the soft X-ray spectrum obtained with $A S C A$ is consistent with emission from two-temperature gas in collisional ionization equilibrium (CIE), which may be associated with nuclear and spatially extended starburst regions seen in the ROSAT HRI data (Wilson et al. 1992). On the other hand, Netzer \& Turner (1997) argue that a combination of warm $\left(T \sim 1.5 \times 10^{5} \mathrm{~K}\right)$ and hot $\left(T \sim 3 \times 10^{6} \mathrm{~K}\right)$ gas in photoionization equilibrium (PIE) is also consistent with the ASCA data. Despite the fact that both models adequately fit the data, their are problems in the inferred parameters that cast doubt on these interpretations. The CIE fit requires a low-temperature component which is essentially metal-free (abundances $\sim 3 \%$ of the solar photosphere). The PIE model requires a steep soft X-ray continuum component (photon index $\Gamma=3.4$ ) that contains a large fraction of the total X-ray luminosity and does not have an obvious physical interpretation. On the other hand, a detailed X-ray spectral analysis of another Seyfert 2 galaxy, Mkn 3, by Griffiths et al. (1998) shows that emission from plasmas both in CIE and PIE can fit the composite Ginga, ASCA, and ROSAT data.

The Circinus Galaxy is the closest ( $D \approx 4 \mathrm{Mpc}$; Freeman et al. 1977) and one of the brightest Seyfert 2 galaxies in the X-ray band. Extensive studies at various wavelengths have provided firm evidence for an obscured Seyfert nucleus at the center of the galaxy; the detection of a prominent one-sided [O III] ionization cone (Marconi et al. 1994), optical and near-IR coronal line emission ${ }^{4}$ from the nuclear region (Oliva et al. 1994; Moorwood et al. 1996), bipolar radio lobes orthogonal to the plane of the galaxy (Elmouttie et al. 1995), rapid variations of $\mathrm{H}_{2} \mathrm{O}$ maser emission from the nucleus (Greenhill et al. 1997), polarized and broad $\mathrm{H} \alpha$ emission from the nucleus (Oliva et al. 1998), and a highly absorbed hard X-ray spectrum with a bright iron $\mathrm{K}$ fluorescent line near $6.4 \mathrm{keV}$ (Matt et al. 1996; Guainazzi et al. 1999). The system is also known to contain regions of starburst activity around the nucleus that account for a large fraction of the bolometric luminosity (Rowan-Robinson \& Crawford 1989). Recent studies of the infrared spectrum indicate that the

\footnotetext{
${ }^{4}$ The term coronal lines here refers to high excitation forbidden lines, which were first observed in the solar corona. These lines can, in principle, be produced either through photoionization or collisional ionization, and are unrelated to emission lines from coronal plasmas, a term which refers to optically thin emission from collisionally ionized plasmas.
} 
relative contributions from starburst and AGN activity, which can be traced through emission from hot dust and ionized gas, respectively, are approximately equal (Maiolino et al. 1998; Genzel et al. 1998).

In a preliminary analysis of the $A S C A$ data by Matt et al. (1996), it was shown that the $2-10$ $\mathrm{keV}$ spectrum is dominated by reflection from cold matter, possibly originating from the inner wall of the putative molecular torus that surrounds the central nucleus. Several emission lines were also detected in the soft X-ray region $(0.5-2 \mathrm{keV})$, most of which were inferred to be present in the lower resolution BeppoSAX spectrum as well (Guainazzi et al. 1999). The measured energies of these emission lines indicate that they are produced through excitation of highly ionized material, and cannot be produced in a cold reflection medium. The soft X-ray region is certainly not dominated by cold reflection.

The purpose of this paper is to perform a detailed analysis of the soft X-ray spectrum of the Circinus Galaxy using various spectral models, and to derive physical parameters that characterize the structure of the X-ray emission line regions. As we show, the soft X-ray spectrum cannot distinguish spectroscopically between collisionally ionized gas and photoionized gas (cf., Netzer, Turner, \& George 1998). There is a strong piece of evidence, however, against an interpretation in terms of collisionally ionized gas, and the inferred parameters somewhat favor photoionization equilibrium. We discuss the implications of our results and investigate the properties of the active nucleus and its circumnuclear environment.

\section{Observation and Data Reduction}

The Circinus Galaxy was observed with $A S C A$ during August $14-15,1995$. For the purposes of our analysis, we only use data obtained by the Solid-state Imaging Spectrometers (SIS), which have the highest spectral resolving power in the soft X-ray band. The SIS data were taken in FAINT and BRIGHT 2-CCD modes, while most of the photons from the Circinus Galaxy were recorded on SIS0-CHIP1 and SIS1-CHIP3. A quick analysis of the SIS0 and SIS1 data indicated a $\sim 1 \%$ offset in the energy scale in the iron-K region, while no obvious offset was observed in the soft X-ray band (although this is most likely due to the poor statistical quality of data in that region). We applied $\mathrm{a} \sim 1 \%$ gain correction to the SIS1-CHIP3 response matrix. Since the spectral resolving power of the GIS is lower by a factor of $\sim 3$ in the soft X-ray region, the discrete features observed in the SIS are smeared out in the GIS. We, therefore, only use data collected on the SIS for our spectral analysis.

The data were screened though the standard criteria using FTOOLS v4.0. After conversion of FAINT mode data to BRIGHT mode data, we combined them with true BRIGHT mode data to obtain a total exposure time of $33 \mathrm{ksec}$ amounting to $\sim 4000$ and $\sim 3000$ counts on the SIS0 and SIS1 detectors, respectively. Although this method does not fully exploit the resolving power capabilities of the SIS, we can significantly improve the statistical quality of the data by compromising for a 20 
- 30\% degredation of the resolving power.

\section{Spectral Analysis}

In this section, we discuss the spectral models that we use for our analysis of the SIS0 data. All of the models we consider here consist of (1) a reflection component, (2) a soft continuum component, and (3) a soft X-ray emission line component. Possible physical interpretations of each of the spectral components are as follows. The reflection component represents a fraction of the intrinsic AGN continuum radiation that is Compton-scattered in a cold medium into our line of sight. The spectrum resembles that of a highly absorbed continuum with a number of K-shell fluorescent lines superimposed (Lightman \& White 1988; George \& Fabian 1991; Matt, Brandt, \& Fabian 1996), which we simply represent by a set of gaussian lines and a Compton reflected powerlaw continuum (i.e., PEXRAV in XSPEC). This component has a number of free parameters; line energies and fluxes of $\mathrm{K}$-shell $\mathrm{Ne}, \mathrm{Mg}, \mathrm{Si}, \mathrm{S}, \mathrm{Ar}, \mathrm{Ca}$, and $\mathrm{Fe}$, the intrinsic powerlaw photon index, and the total reflected flux. We assume that the K-shell fluorescent lines are infinitely narrow, which is justified by the observed $\mathrm{Fe} \mathrm{K} \alpha$ line profile, as we discuss later. The soft continuum component may be produced through electron scattering of the primary radiation in the surrounding gas, in which case the photon index should be equal to that of the primary radiation, or through inverse Compton scattering of infrared photons with relativistic electrons. For the latter case, the photon index should match the photon index in the radio band. The soft X-ray emission line component is modeled as either a collisionally ionized or a photoionized plasma in equilibrium. We assume that the entire spectrum is absorbed by a single column density (Galactic + local absorption). The reflection component possibly suffers higher absorption, since the reflection region (the inner wall of the molecular torus) is assumed to be located closer to the nucleus. The reflected spectrum, however, is not sensitive to $N_{H} \lesssim 10^{22} \mathrm{~cm}^{-2}$ since most of the flux is contained in the region above $\sim 2 \mathrm{keV}$ anyway. Throughout this paper, we adopt a distance of $4 \mathrm{Mpc}$ to the Circinus Galaxy.

\subsection{Collisional Ionization Equilibrium Model}

We first adopt a model in which the soft X-ray component is represented by a single temperature thermal plasma in collisional ionization equilibrium (i.e., MEKAL in XSPEC), with the temperature, overall abundance, and the emission measure free parameters in the fit. For the reflection component, we first allowed the fluorescent line energies to be free, and found that the model is unable to locate the centroid energies for all but the $\mathrm{Fe} \mathrm{K} \alpha$ and $\mathrm{K} \beta$ lines. We, therefore, froze the energies of all of the fluorescent lines, except for those of iron, at values shown in Table 1, which are those derived from the photoionization equilibrium fit discussed in $\S 3.2$. These are more or less consistent with the ionization state inferred from the iron $\mathrm{K} \alpha$ line energy (i.e., partially ionized medium), which is also consistent with the iron $\mathrm{K} \alpha$ line energy measured with BeppoSAX (Guainazzi et al. 1999). The measured fluorescent line intensities are also listed in Table 1. Con- 
trary to Matt et al. (1996), we find a statistically acceptable fit with $\chi_{r}^{2}=1.21$ for 305 degrees of freedom. The best-fit model in comparison with the data is shown in Figure 1 and the derived parameters are listed in Table 2 .

The allowed ranges in temperature and emission measure $\left(E M=\int n_{e}^{2} d V\right)$ are shown in Figure 2 where we plot 68, 90, and $99 \%$ confidence ranges for two interesting parameters. We obtain $90 \%$ confidence ranges of $k T=0.69_{-0.07}^{+0.05} \mathrm{keV}$ and $E M=\left(1.12_{-0.10}^{+0.17}\right) \times 10^{63} \mathrm{~cm}^{-3}$. Including an additional thermal component does not improve the fit significantly $\left(\Delta \chi^{2} \sim 1\right)$. In fact, if we adopt a powerlaw differential emission measure (DEM) model where the emission measure as a function of $k T$ is proportional to $(k T)^{\alpha}$, we obtain a steep slope of $\alpha \sim 5$ and a sharp hightemperature cutoff at $k T \sim 0.7 \mathrm{keV}$, which nearly corresponds to a single temperature model at $k T \sim 0.7 \mathrm{keV}$. Similar results are obtained for different DEM models in coronal equilibrium. In any case, the total emission measure in the thermal component is $\sim 1 \times 10^{63} \mathrm{~cm}^{-3}$ and the corresponding unabsorbed luminosity is $L_{x}=3 \times 10^{40} \mathrm{erg} \mathrm{s}^{-1}$.

\subsection{Photoionization Equilibrium Model}

We next test a model in which the soft X-ray emission lines are produced through cascades following radiative recombination in photoionization equilibrium. This spectral model was originally constructed for the analysis of the ASCA spectrum of Cygnus X-3 (Liedahl \& Paerels 1996) and was also succesfully applied to the $A S C A$ data of Vela X-1 (Sako et al. 1999). The model includes line emission from hydrogen- and helium-like carbon, nitrogen, oxygen, neon, magnesium, silicon, sulfur, argon, and calcium, as well as the highest ten charge states of iron (Fe XVII - Fe XXVI), and the associated radiative recombination continua (RRC). For a detailed description of the atomic model, see Sako et al. (1999).

We first start by fitting the data with the recombination cascade model, using only the Kshell ions of oxygen, neon, magnesium, silicon, sulfur, argon, calcium, and iron, to construct an empirical DEM. Due to the limited statistical quality of the data, we cannot directly constrain the temperature of each ion emission region from the shape of the RRC, so we fix the temperature of each ion at their respective temperature of formation. We do this by first calculating the ionization balance and temperature as functions of the ionization parameter $\left(\xi=L_{x} /\left(n_{p} r^{2}\right)\right.$, where $L_{x}$ is the X-ray luminosity between 1 and 100 Rydbergs, $n_{p}$ is the proton number density, and $r$ is the distance from the ionizing source; Tarter, Tucker, \& Salpeter 1969) using the photoionization code, XSTAR (Kallman \& Krolik 1995). We assume that the medium is optically thin with respect to continuum absorption, which is an excellent approximation, as we show later. We adopt an exponential cutoff powerlaw ionizing continuum with a photon index of $\Gamma=1.7$ with an e-folding energy of $E_{f}=100 \mathrm{keV}$ and a cutoff energy of $E_{c}=60 \mathrm{keV}$ (Guainazzi et al. 1999). Using these results, we then calculate the temperature at which each of the ion line emissivities is a maximum and fix each ionic temperature at those values, which we refer to as the temperatures of formation. This is a good first order approximation since a large fraction of the total ion line fluxes 
are produced near those temperatures. Approximately $50 \%$ of the recombination line emissivity of the He-like ions originates from a range in $\Delta \log \xi$ of $\sim 0.5$. For the $\mathrm{H}$-like ions, the width is broader $(\Delta \log \xi \sim 1)$ since the emission lines are formed through recombination onto a bare nucleus, which exists in a wider range in ionization parameter.

We fit the SIS0 and SIS1 data simultaneously and obtain a statistically acceptable fit with $\chi_{r}^{2}=1.10$ with 290 degrees of freedom. The data in comparison to the model are plotted in Figure 3. In addition to the continuum parameters, this model contains normalizations of each ion line flux, which are proportional to their ion line emission measures. Figure 4 shows the derived empirical DEM distribution assuming an emission width of $\Delta \log \xi \sim 0.5$ for helium-like ions and $\Delta \log \xi \sim 1$ for the hydrogenic species for two sets of assumed chemical abundances; (1) solar photospheric abundances (Anders \& Grevesse 1989) and (2) abundances derived by Oliva, Marconi, \& Moorwood (1999) for a knot located approximately $15^{\prime \prime}$ from the nucleus in the direction of the [O III] cone. We adopt solar abundances for elements that are not listed in Oliva, Marconi, \& Moorwood (1999). Given the assumptions that we have made in the construction of the empirical DEM distribution, we estimate the points drawn on Figure 4 to be uncertain by up to a factor of 2 $-3$.

With the exception of the DEM points derived from O VII and O VIII at $\log \xi=1.4$ and $\log \xi=1.9$, respectively, the shape of the empirical DEM is nearly linear with evidence of a slight decrease in DEM with increasing $\log \xi$. The oxygen ions have bright features below $E \sim 1 \mathrm{keV}$ and the derived emission measures are extremely sensitive to the foreground column density. An increase in the column density by $50 \%$ can be offset by an increase in the emission measure of $\mathrm{O}$ VII by a factor of $\sim 10$ and O VIII by a factor of $\sim 6$. Keeping this in mind, the derived DEM distribution, to first order, is nearly linear.

We then adopt a reverse fitting procedure where we parametrize the model spectrum of the highly ionized component according to powerlaw DEMs of the form, $d(E M) / d \log \xi \propto \xi^{\gamma}$, with minimum and maximum cutoff ionization parameters, $\xi_{\min }$ and $\xi_{\max }$, respectively. This procedure bypasses the ambiguities in the assumed temperatures of formation and correctly takes account of the temperature gradient in each ionization zone. In addition to the K-shell ions that we had initially used in the empirical fit, this time we also include line and RRC emission from the iron $\mathrm{L}$ species, which are expected to contribute $\sim 10 \%$ to the total recombination emission flux. There are four free parameters in this component, namely, the slope $\gamma$ of the DEM as a function of ionization parameter, the normalization, which is proportional to the total emission measure $E M$, low- $\xi$ cutoff $\xi_{\min }$, and high- $\xi$ cutoff $\xi_{\max }$. Unlike the CIE case, we are able to centroid the fluorescent line energies of all the elements that we list in Table 2, and we find a statistically acceptable fit with $\chi_{r}^{2}=1.12$ for 299 degrees of freedom. The data in comparison to the model is shown in Figure 5 and the best-fit parameters are listed in Table 3. We also derive confidence contours for two pairs of interesting parameters; $\left(\xi_{\min }, \xi_{\max }\right)$ and $(\gamma, E M)$, as shown in Figures 6 and 7 . In summary, we derive $90 \%$ confidence ranges of $\gamma=-0.07_{-0.14}^{+0.12}$ and $E M=(5.5 \pm 0.7) \times 10^{63} \mathrm{~cm}^{-3}$ for two interesting parameters, and $\log \xi_{\min }=0.91_{-\infty}^{+0.48}$ and $\log \xi_{\max }=3.53_{-0.20}^{+0.32}$, again, for two interesting 
parameters. The best-fit model spectrum with the various components is shown in Figure 8 .

The lower limit on $\xi_{\min }$ cannot be well-constrained simply because the X-ray spectrum of the Circinus Galaxy is absorbed through a moderately high column density $\left(N_{H} \sim 8 \times 10^{21} \mathrm{~cm}^{-2}\right)$, and also because the $A S C A$ SIS detectors are not sensitive to X-rays below $\sim 0.5 \mathrm{keV}$, where discrete recombination emission from regions with $\log \xi \sim 1$ dominates, for example, from carbon and nitrogen. The upper limit on $\xi_{\max }$ is constrained mainly by the Ly $\alpha$ flux of Fe XXVI.

\section{Discussion and Implications}

We have shown that the soft X-ray emission line spectrum of the Circinus Galaxy is compatible with both coronal and photoionization equilibrium, and the excitation mechanisms cannot be spectroscopically distinguished without any ambiguity. In this section, we extract physical parameters implied by each model and discuss their validity in relation to observations at other wavelength bands.

According to recent ROSAT and ASCA observations, nearby star-forming galaxies show Xray spectra that can be well described by a combination of a soft optically thin thermal plasma with a temperature of $k T \sim 0.6 \mathrm{keV}$ and a hard continuum component with either a photon index of $\Gamma \sim 1.7$ or a bremsstrahlung temperature of $k T \sim 5 \mathrm{keV}$ (e.g., Ptak et al. 1999, and references therein). The collisional ionization interpretation for the Circinus Galaxy gives a best-fit temperature of $\sim 0.69 \mathrm{keV}$ and a luminosity of few $\times 10^{40} \mathrm{erg} \mathrm{s}^{-1}$ in this component, which are typical values for starburst galaxies. Note that the temperature of the plasma is well-constrained and that the data do not allow the presence of gas with temperature lower than $k T \sim 0.60 \mathrm{keV}$ at the $99 \%$ confidence level. It is interesting to compare this with what Oliva et al. (1994) have found using visible and near infrared observations of the coronal emission lines in the Circinus Galaxy. In order to reproduce the observed spectrum in the context of a powerlaw DEM plasma in CIE, they require a sharp cutoff in the DEM at a temperature of $k T_{\max } \sim 0.1 \mathrm{keV}$. This would imply that there is a gap in the emission measure distribution in the range $0.1 \lesssim k T \lesssim 0.6 \mathrm{keV}$, which has no obvious physical interpretation.

In PIE, the assumption of plasma heating by a point source of X-radiation places an additional constraint on the plasma structure (e.g., the heating rate as a function of $\xi$ ), which can be then used to derive the geometrical distribution of the gas. In the PIE interpretation of the spectrum, have shown that a nearly flat DEM distribution is consistent with the data. One simple way of producing a flat DEM curve is to adopt a radial density profile of the form, $n_{e}(r) \propto r^{-3 / 2}$. This is perhaps the simplest configuration but, by no means, unique. More generally, a powerlaw density profile produces a powerlaw DEM curve. It is interesting to note, however, that a conical homogeneous medium with a powerlaw density profile as a function of radius always produces a powerlaw DEM distribution; i.e., a simple calculation shows that for a density profile of the form,

$$
n_{e}(r)=n_{e 0} r_{\mathrm{pc}}^{-\beta}
$$


where we define $n_{e 0}$ to be the density in $\mathrm{cm}^{-3}$ at a distance of $1 \mathrm{pc}$ from the central source, the DEM distribution can be written as, $d(E M)=\Delta \Omega n_{e}^{2} r^{2} d r$, or

$$
\frac{d(E M)}{d(\log \xi)}=\ln 10 \frac{\Delta \Omega}{|\beta-2|}\left[n_{e 0}\left(\mu L_{x}\right)^{3-2 \beta}\right]^{1 /(2-\beta)} \xi^{(3-2 \beta) /(\beta-2)},
$$

where $\mu$ is the ratio of the electron to proton number densities ( $\mu=1.2$, for an X-ray emitting plasma) and $\Delta \Omega$ is the opening solid angle of the photoionized region. From this equation, we can identify the slope of the DEM curve $\gamma$ to be $(3-2 \beta) /(\beta-2)$. Our inferred range in $\gamma$ corresponds to $\beta=1.48_{-0.04}^{+0.03}$. One can also see that $\beta=3 / 2$ corresponds to a flat DEM curve.

The fact that $\beta$ is relatively well-constrained is due to the fact that the slope of the DEM curve is extremely sensitive to the value of $\beta$. As mentioned earlier, a $\beta=3 / 2$ density profile produces a flat DEM curve, which produces emission lines and RRC from a wide range of ionization stages. A $\beta=2$ profile, on the other hand, corresponds to a single- $\xi$ zone, since both the ionizing flux and the density decay like $\propto r^{-2}$. In this case, only a small number of charge states can exist, and the resulting spectrum is more sparse.

Our derived values for the parameters shown in Table 4 depend on the assumed metal abundances. If we assume that the abundances are $A_{Z_{\odot}}$ relative to solar, the DEM curve will be uniformly shifted by a factor of $A_{Z_{\odot}}^{-1}$ and the inferred total emission measure will be $E M=$ $5.5 \times 10^{63} A_{Z_{\odot}}^{-1} \mathrm{~cm}^{-3}$. The parameters, $\beta, \xi_{\min }$, and $\xi_{\max }$ are unaffected by a uniform shift in abundances, since they characterize only the shape of the DEM curve and not its normalization. The exact dependences of these parameters on the abundance of an individual element, however, is more complex. For example, an underabundance of oxygen and neon, as found by Oliva, Marconi, \& Moorwood (1999), may result in a steeper DEM slope $(\beta \gtrsim 1.5)$ or a decrease in $\xi_{\text {min }}$ in order to increase the emission measure at low- $\xi$ and compensate for the observed flux at low energies.

Although the density parametrization of equation 1 is simplistic, chosen for the photoionization interpretation of the spectrum, there is empirical evidence that narrow-line regions in many Seyfert galaxies, in fact, do exhibit this type of behavior. For $\beta=2$, the density scales like $r^{-2}$, and, hence, the ionization parameter is constant with radial distance. This has been observed in some systems that show correlations between widths of optical emission lines and their critical densities for collisional de-excitation. Spectral transitions with higher critical densities generally exhibit broader line profiles. There are also cases where the observed widths are higher for transitions in ions with higher ionization potential, which corresponds to a scenario with $\beta<2$ (e.g., Pelat, Alloin, \& Fosbury 1981; Filippenko \& Halpern 1994; De Robertis \& Osterbrock 1984). According to De Robertis \& Osterbrock (1986), values of $\beta$ lie between 0 and 2, while more Seyfert 2 galaxies have $\beta$ closer to 2 . A $\beta>2$ behavior (i.e., $\xi$ increasing with distance) is not observed in any Seyfert galaxy.

Using the empirical density profile, we can estimate several physical parameters from the results of the DEM fit. From the best-fit values for $\beta$ and the total $E M$, which is proportional to the prefactor in equation $2, \Delta \Omega\left(n_{e 0} L_{x}^{3-2 \beta}\right)^{1 /(2-\beta)}$, we estimate the density profile of the X-ray 
emission region to be,

$$
n_{e}(r)=4.0 \times 10^{2} \Delta \Omega^{-0.52} L_{x(42)}^{-0.04} r_{\mathrm{pc}}^{-1.48}\left(\mathrm{~cm}^{-3}\right),
$$

where $L_{x(42)}$ is the X-ray luminosity in multiples of $10^{42} \mathrm{erg} \mathrm{s}^{-1}$. We also saw that the upper and lower limits on the ionization parameter were $\log \xi_{\max } \sim 3.5$ and $\log \xi_{\min } \lesssim 0.9$, respectively. From the upper limit, we can estimate the distance from the central nucleus to the innermost X-ray emission line region that is directly visible from the observer. We estimate the inner distance to be $r_{\text {in }} \sim 0.011 \Delta \Omega L_{x(42)}^{2}$ pc. Similarly, the lower limit on the outer distance is $r_{\text {out }} \sim 1200 \Delta \Omega L_{x(42)}^{2}$ pc. We can see from the [O III] image that the opening half-angle of the one-sided ionization cone is $\sim 30^{\circ}$, which corresponds to a solid angle of $\Delta \Omega \sim 0.8$. Assuming that the X-ray emission lines are produced in the inner region close to the base of this ionization cone, we estimate its size to be $\sim 900$ pc or approximately $50^{\prime \prime}$ in the sky. This is qualitatively consistent with the ROSAT HRI image that shows a slight spatial extent $\left(\lesssim 30^{\prime \prime}\right)$ approximately in the direction of the $[\mathrm{O}$ III $]$ cone.

As a cross-check on the derived parameters, we calculate the electron scattering optical depth through the emission line medium and compare it with the observed scattered continuum flux. As listed in Table 3, the unabsorbed luminosity of the scattered continuum is $L_{x}^{\text {scat }} \sim 8.4 \times 10^{39} \mathrm{erg} \mathrm{s}^{-1}$, which corresponds to a Thomson optical depth of $\tau_{\mathrm{es}}=0.084\left(\Delta \Omega L_{x(42)}\right)^{-1}$. Integrating the density profile (equation 3) from $r_{\text {in }}$ out to $r_{\text {out }}$ yields an optical depth of, $\tau_{\text {es }}=0.014\left(\Delta \Omega L_{x(42)}\right)^{-1}$. We note that regions beyond $r_{\text {out }}$ do not contribute significantly to the optical depth. This implies the presence of additional scattering regions in the circumsource medium, which must be either more highly ionized or less ionized than the X-ray emission line region. The additional column density required is $N_{H}(\Delta \Omega / 4 \pi) \sim 1 \times 10^{23} \mathrm{~cm}^{-2}$, which may be produced in the region that absorbs the transmission component observed in the hard X-rays. The measured column density of this component is $N_{H} \sim 4 \times 10^{24} \mathrm{~cm}^{-2}$ (Guainazzi et al. 1999), and thus a covering fraction of $(\Delta \Omega / 4 \pi) \sim 0.03$ can produce most of the observed soft continuum flux. An optical depth of $\tau_{\text {es }} \sim$ 0.01 through the photoionized gas amounts to a total column density of $N_{H} \sim$ few $\times 10^{21} \mathrm{~cm}^{-2}$, which justifies our assumption that the primary radiation is optically thin to continuum absorption.

It is interesting to note that a density profile of the form $n(r) \sim r^{-3 / 2}$ is expected for Bondi accretion in a spherically symmetric gravitational potential of a point source. For a central black hole mass of $M_{6}$ (in multiples of $10^{6} M_{\odot}$ ), the gas is nearly in free-fall within an accretion radius of $r_{\text {acc }} \sim 100 M_{6} T_{\infty(4)}^{-1}$ pc, where $T_{\infty(4)}$ is the ambient gas temperature (in multiples of $10^{4} \mathrm{~K}$ ) far away from the central compact object. Using the density profile of equation 3 and a free-fall velocity profile (i.e., $v \propto r^{-1 / 2}$ ), the mass inflow rate can be expressed as,

$$
\dot{M}_{\text {inflow }}=8 \times 10^{-4} \Delta \Omega^{0.48} L_{x(42)}^{-0.04} M_{6}^{1 / 2}\left(M_{\odot} \mathrm{yr}^{-1}\right) .
$$

The mass accretion rate implied by the observed X-ray luminosity is,

$$
\dot{M}_{\text {accretion }}=2 \times 10^{-4} L_{x(42)} \eta_{0.1}^{-1}\left(M_{\odot} \mathrm{yr}^{-1}\right),
$$


where $\eta_{0.1}$ is the energy conversion efficiency in multiples of 0.1 . Since the accretion radius is on the order of or larger than the size of the X-ray emission line region, the outer region may not necessarily be inflowing at the free-fall velocity, especially since photoelectric heating by the AGN continuum radiation increases the local sound speed of the surrounding gas. Nevertheless, these mass flow rates are roughly consistent with each other and we suggest that the inner part of the X-ray emission region is fueling the central engine. Projected velocity shifts of up to $\sim 230 \mathrm{~km} \mathrm{~s}^{-1}$ have also been detected near the base of the [O III] and $\mathrm{H} \alpha$ ionization cones, which may be interpreted as either an outflow or inflow relative to the nucleus (Veilleux \& Bland-Hawthorn 1997; Elmouttie et al. 1998).

We suggest that the region that produces the observed X-ray recombination lines is the confining medium of the so-called coronal line regions (CLR; Oke \& Sargent 1968). Using the surface brightness of [Fe XI], (Oliva et al. 1994) finds the CLR to have a size $\sim 0.5^{\prime \prime}$ (diameter of $\sim 10 \mathrm{pc}$ ) and a gas density of $n_{e} \sim 250 f^{-1 / 2} \mathrm{~cm}^{-3}$, where $f$ is the volume filling factor. The temperature is assumed to be $k T \sim 3 \times 10^{4} \mathrm{~K}$, a typical value for a photoionized CLR. Using the density profile inferred from our fit, we estimate the gas density at a distance of $\sim 10$ pc to be $n_{e} \sim 10 \mathrm{~cm}^{-3}$. According to our model, the ionization parameter at this distance is $\log \xi \sim 2.5$ with a temperature of $k T \sim 2 \times 10^{6} \mathrm{~K}$, and thus the pressure is $n_{e} T \sim 2 \times 10^{7}$. In order for the two regions to be in pressure equilibrium, the filling factor of the CLR must be $f \sim 0.1$. This implies the density of the [Fe XI] region to be $n_{e} \sim 800 \mathrm{~cm}^{-3}$, which is still much lower than the critical density of the upper level and, therefore, the line will not be diminished.

The model that we have suggested can be further tested with high spectral and spatial resolution grating observations with the Chandra observatory. Our model predicts that $\sim 70 \%$ of the total flux will be spatially unresolved, containing the reflection component, as well as a large fraction of the scattered soft continuum component. The remaining $\sim 30 \%$, which is dominated by recombination line emission, will be extended. Specifically, recombination lines from low- $Z$ ions (e.g., $\mathrm{O}, \mathrm{Ne}$, and $\mathrm{Mg}$ ) will be extended by $\sim 10^{\prime \prime}$ and those from higher- $Z$ ions (e.g., Si, S, Ar, $\mathrm{Ca}$, and $\mathrm{Fe}$ ) will have a spatial extent of $\lesssim 5^{\prime \prime}$. In addition to the spatial distibution of the X-ray emission line region, measurements of velocity shifts will allow us to constrain the kinematics and determine whether the confining medium is flowing towards or away from the central nucleus.

This work has benefited from useful discussions with Andy Rasmussen concerning the $A S C A$ SIS calibrations. We have made use of data obtained through the High Energy Astrophysics Science Archive Research Center Online Service, provided by the NASA/Goddard Space Flight Center. We thank the ASCA team and the HEASARC Online Service for their support. This work was supported under a NASA Long Term Space Astrophysics Program grant (NAG5-3541). D. A. L. was supported in part by a NASA Long Term Space Astrophysics Program grant (S-92654-F). Work at LLNL was performed under the auspices of the U. S. Department of Energy, Contract No. W-7405-Eng-48. 


\section{REFERENCES}

Anders, E., \& Grevesse, N. 1989, Geochim. Cosmochim. Acta, 53, 197

Antonucci, R. R. J. 1993, ARA\&A, 31, 473

De Robertis, M. M. \& Osterbrock, D. E. 1984, ApJ, 286, 171

De Robertis, M. M. \& Osterbrock, D. E. 1986, ApJ, 301, 727

Elmouttie, M., Haynes, R. F., Jones, K. L., Ehle, M., Beck, R., \& Wielebinski 1995, MNRAS, 275, L53

Elmouttie, M., Koribalski, B., Gordon, S., Taylor, K. Houghton, S., Lavezzi, T., Haynes, R., \& Jones, K. 1998, MNRAS, 297, 49

Filippenko, A. V. \& Halpern, J. P. 1984, ApJ, 285, 458

Freeman, K. C., Karlsson, B., Lyngå, G., Burrell, J. F., van Woerden, H., Goss, W. M., \& Mebold, U. 1977, A\&A, 445

Genzel, R., et al. 1998, ApJ, 498, 579

George, I. M. \& Fabian, A. C. 1991, MNRAS, 249, 352

Greenhill, L. J., Ellingsen, S. P., Norris, R. P., Gough, R. G., Sinclair, M. W., Moran, J. M., \& Mushotzky 1997, ApJ, 474, L103

Griffiths, R. G., Warwick, R. S., Georgantopoulos, I., Done, C., \& Smith, D. A. 1998, MNRAS, 298,1159

Guainazzi et al. 1999, MNRAS, 310, 10

Kallman, T. R., \& Krolik, J. H. 1995, XSTAR - A Spectral Analysis Tool, HEASARC (NASA/GSFC, Greenbelt)

Liedahl, D. A., \& Paerels, F. 1996, ApJ, 468, L33

Lightman, A. P. \& White, T. R. 1988, ApJ, 335, 57

Maiolino, R., Krabbe, A., Thatte, N., \& Genzel, R. 1998, ApJ, 493, 650

Marconi, A., Moorwood, A. F. M., Origlia, L., \& Oliva, E. 1994, The Messenger, 78, 20

Matt, G., Brandt, W. N., \& Fabian, A. C. 1996, MNRAS, 280, 823

Matt, G., et al. 1996, MNRAS, 281, L69

Matt, G., et al. 1999, A\&A, 341, L39 
Moorwood, A. F. M., Lutz, D., Oliva, E., Marconi, A., Netzer, H., Genzel, R., Sturm, E., \& de Graauw, Th. 1996, A\&A, 315, L109

Netzer, H. \& Turner, T. J. 1997, ApJ, 488, 694

Netzer, H., Turner, T. J., \& George, I. M. 1998, ApJ, 504, 680

Oke, J. B., \& Sargent, W. L. W. 1968, ApJ, 151, 807

Oliva, E., Salvati, M., Moorwood, A. F. M., \& Marconi, A. 1994, A\&A, 228, 457

Oliva, E., Marconi, A., Cimatti, A., \& di Serego Alighieri, S. 1998, A\&A, 329, L21

Oliva, E., Marconi, A., \& Moorwood, A. F. M. 1999, A\&A, 342, 87

Otani, C., et al. 1996, PASJ, 48, 211

Pelat, D., Alloin, D., \& Fosbury, A. E. 1981, MNRAS, 195, 787

Ptak, A., Serlemitsos, P., Yaqoob, T., \& Mushotzky, R. 1999, ApJS, 120, 179

Rowan-Robinson, M., \& Crawford, J. 1989, MNRAS, 238, 523

Sako, M., Liedahl, D. A., Kahn, S. M., \& Paerels, F. 1999, ApJ, 525, 921

Tarter, C. B., Tucker, W. H., \& Salpeter, E. E. 1969, ApJ, 156, 943

Ueno, S., Mushotzky, R. F., Koyama, K., Iwasawa, K., Awaki, H., \& Hayashi, I. 1994, PASJ, 46, L71

Veilleux, S., \& Bland-Hawthorn, J. 1997, ApJ, 479, L105

Wilson, A. S., Elvis, M., Lawrence, A., \& Bland-Hawthorn, J. 1992, ApJ, 391, L75 


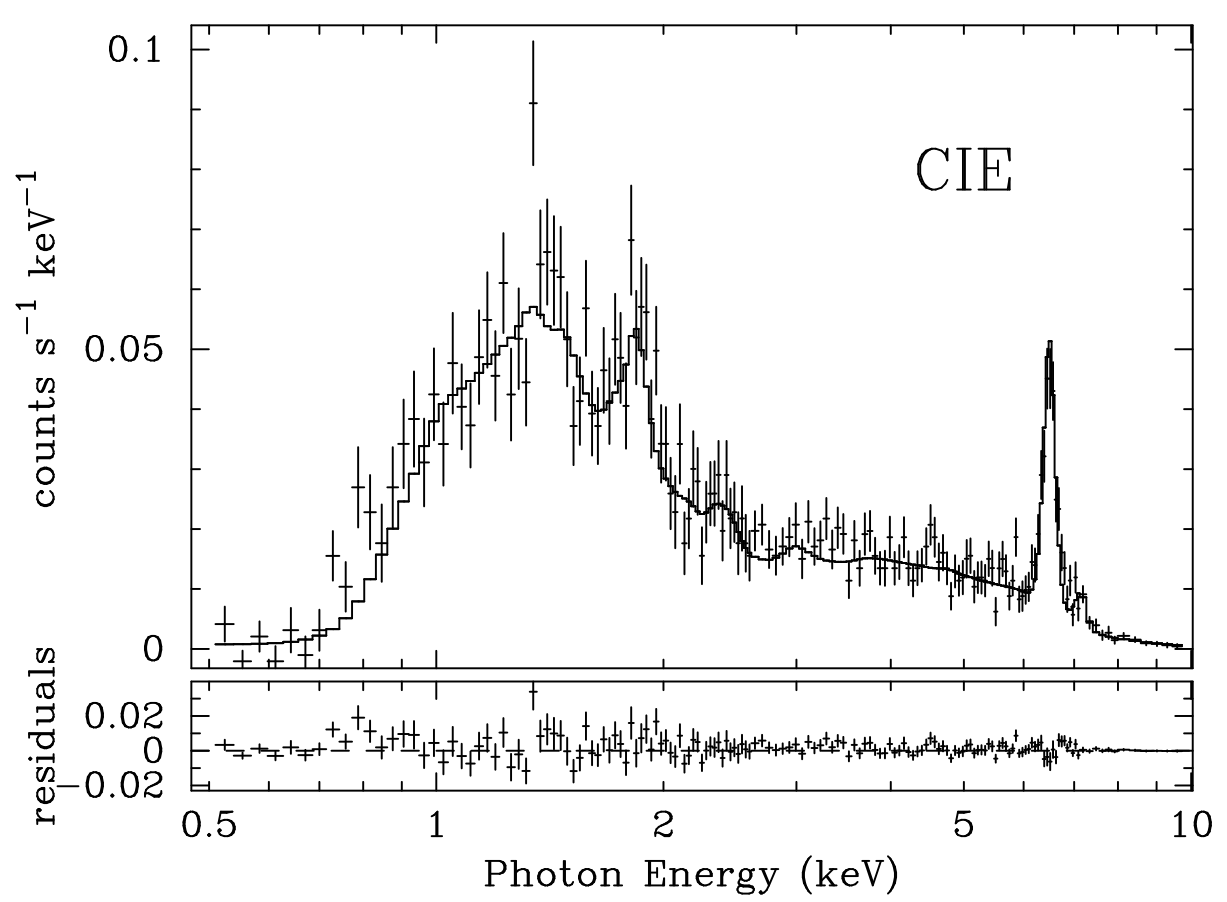

Fig. 1. - The ASCA SIS data and the best-fit CIE model. The fit is statistically acceptable with $\chi_{r}^{2}=1.21$ for 305 degrees of freedom. Only SIS0 data is shown for clarity.

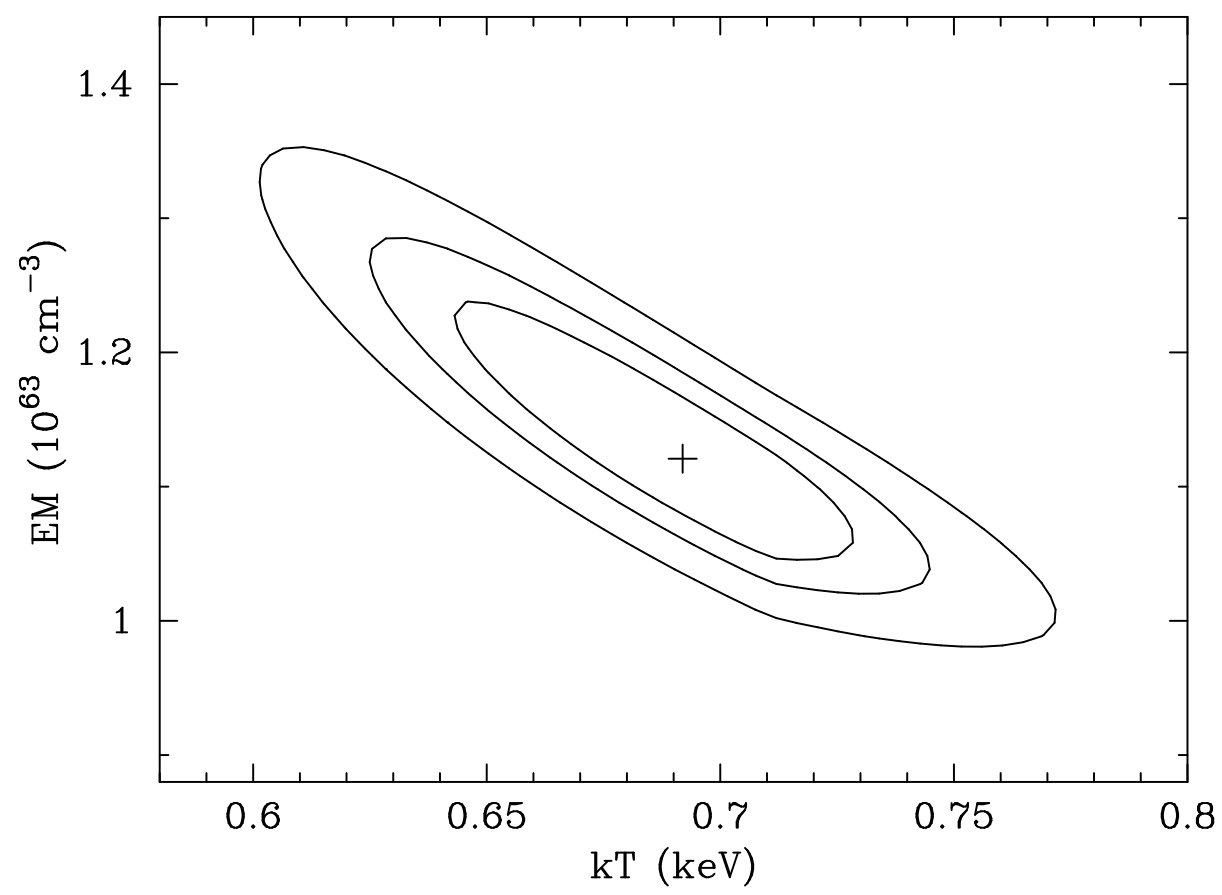

Fig. 2.- Two parameter confidence ranges ( $k T$ vs. total $E M$ ) for the highly ionized component in the CIE fit. Solar abundances are assumed. 


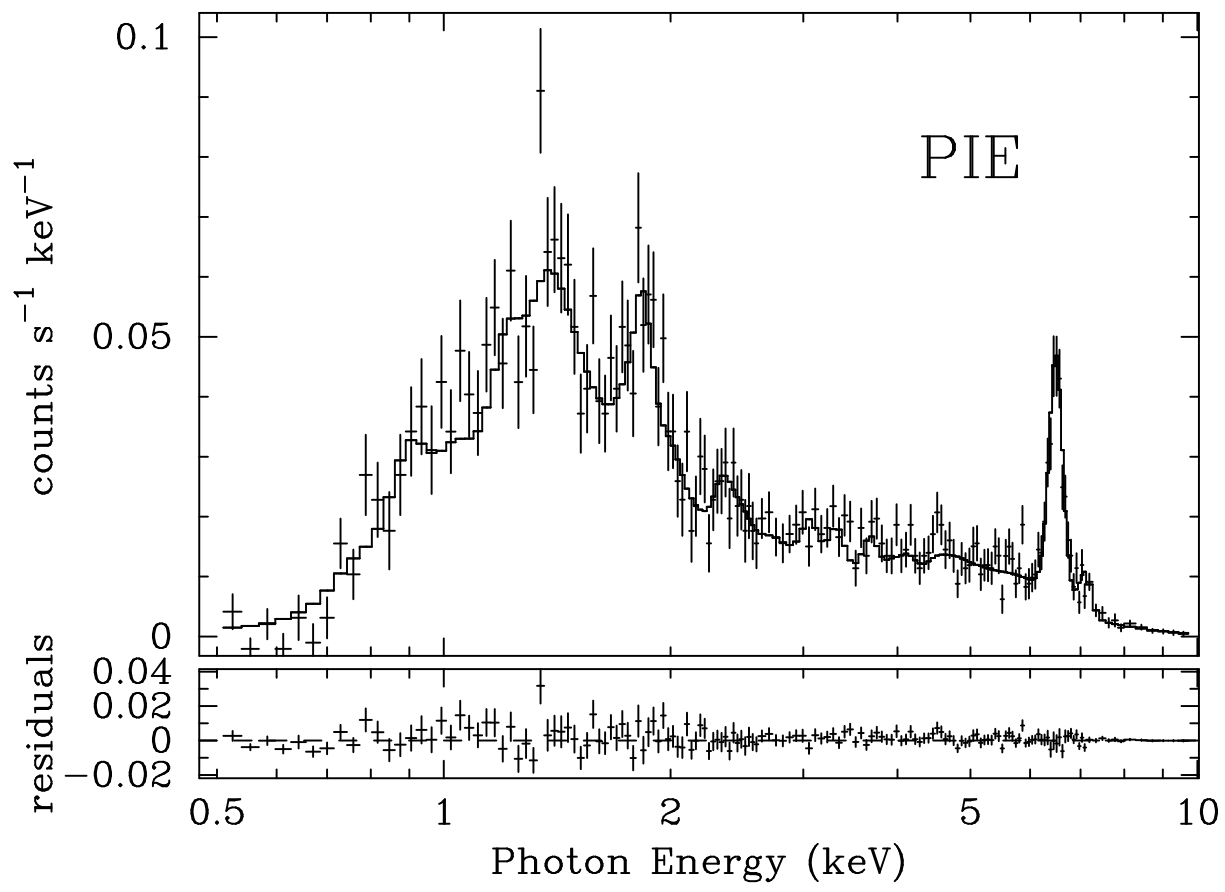

Fig. 3. - The best-fit recombination cascade model compared with the SIS data $\left(\chi_{r}^{2}=1.10\right.$ with 290 degrees of freedom). Again, only SIS0 data is shown for clarity. 


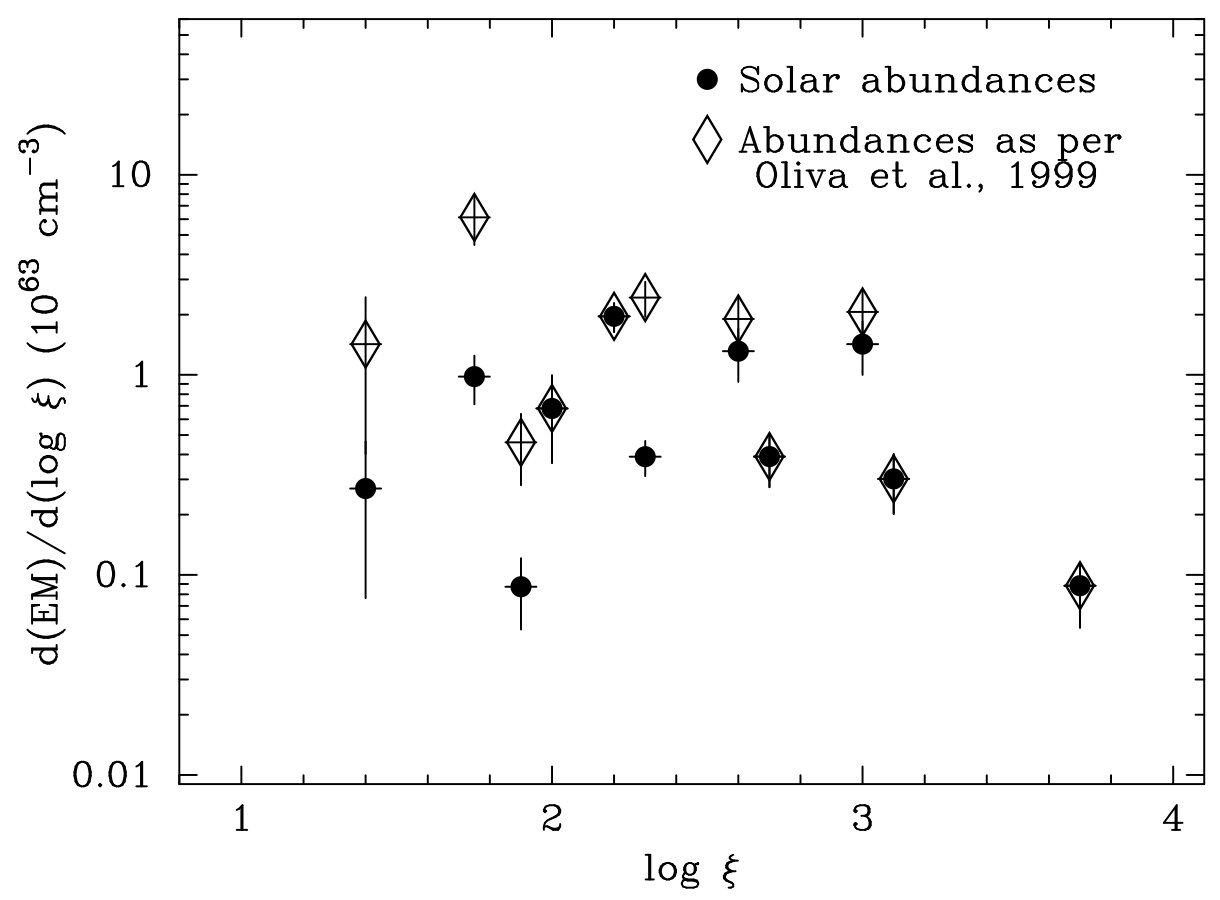

Fig. 4.- An empirical DEM distribution derived from K-shell oxygen, neon, magnesium, silicon, argon, and iron for two sets of assumed chemical abundances; solar photospheric abunandes of Anders \& Grevesse (1989) and abundances derived by Oliva, Marconi, \& Moorwood (1999) for a knot located near the nucleus. 


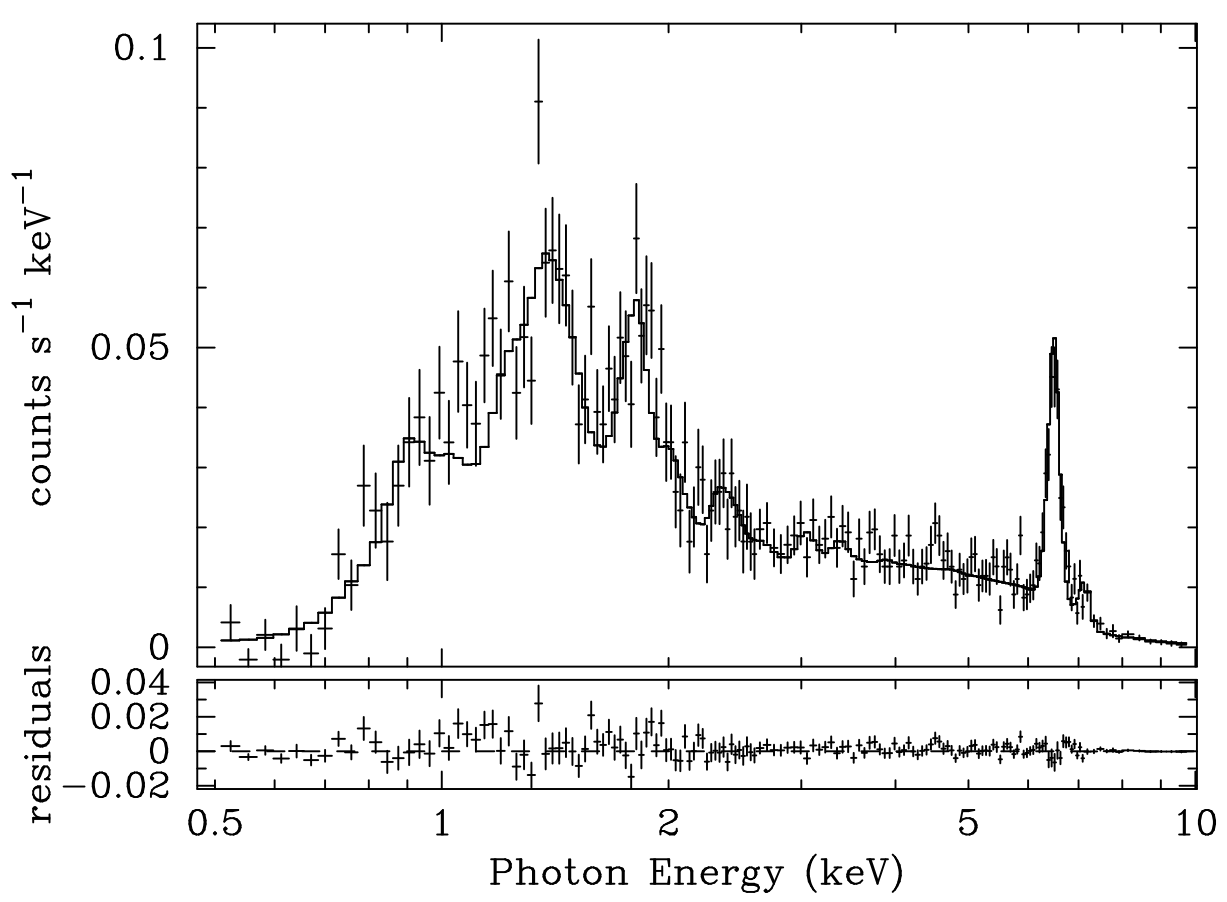

Fig. 5. - The best-fit powerlaw DEM PIE model in comparison to the data. Again, the fit is acceptable with $\chi_{r}^{2}=1.12$ for 299 degrees of freedom.



Fig. 6. - Two parameter confidence ranges for the slope of the DEM curve, $\gamma$, versus the total emission measure, EM, for the PIE DEM fit. 


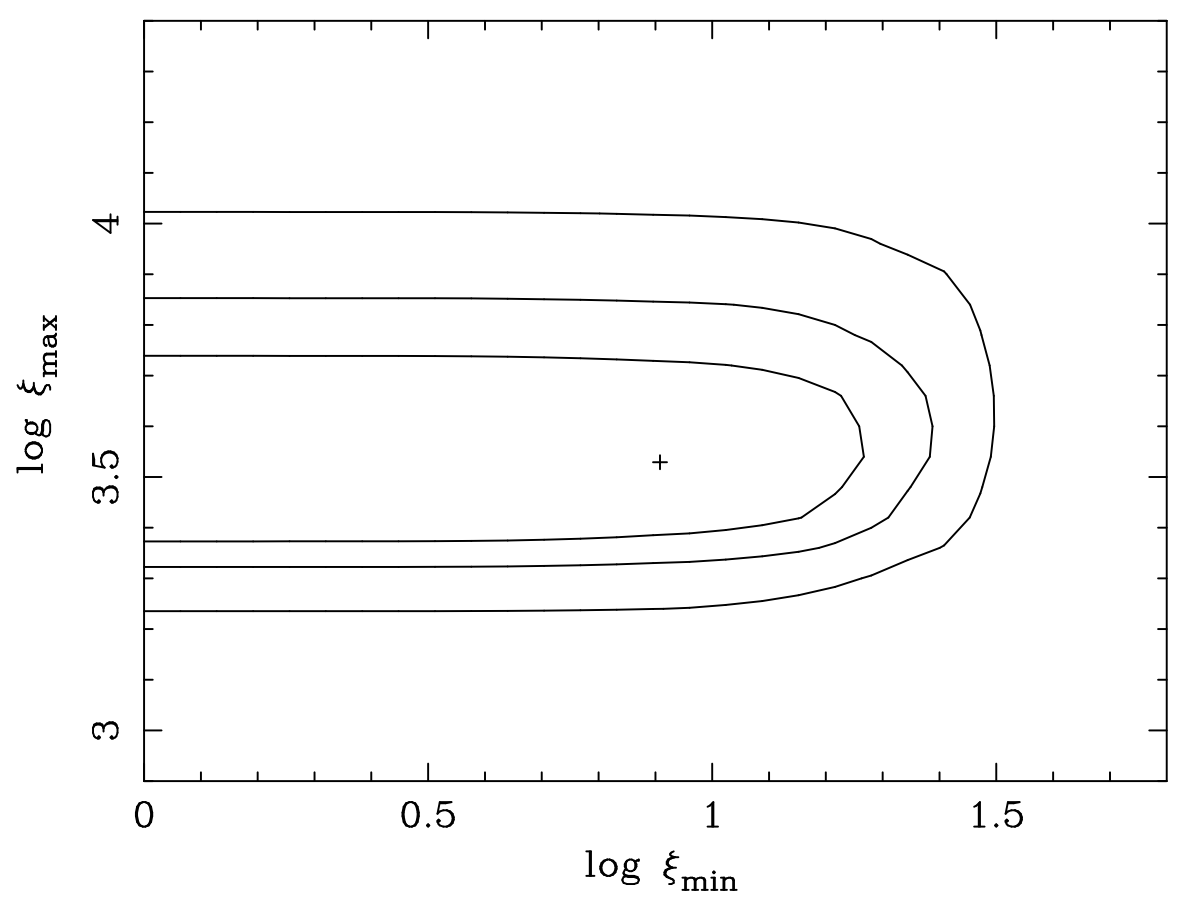

Fig. 7.- Two parameter confidence ranges for the lower and upper cutoff ionization parameters $\left(\log \xi_{\min }\right.$ vs. $\left.\log \xi_{\max }\right)$ for the PIE DEM fit.



Fig. 8.- The best-fit PIE DEM model spectrum for the parameters shown in Table 4. 
Table 1. Summary of Fluorescent Line Intensities

\begin{tabular}{|c|c|c|c|c|c|c|}
\hline \multirow[b]{2}{*}{ Element } & \multirow[b]{2}{*}{ Line Energy ${ }^{b}(\mathrm{keV})$} & \multicolumn{2}{|c|}{ CIE Interpretation $^{\mathrm{a}}$} & \multicolumn{2}{|c|}{ PIE Interpretation ${ }^{\mathrm{a}}$} & \multirow[b]{2}{*}{ Reflection $^{\mathrm{d}}$} \\
\hline & & Line Intensity ${ }^{\mathrm{c}}$ & $\Delta \chi^{2}$ & Line Intensity ${ }^{\mathrm{c}}$ & $\Delta \chi^{2}$ & \\
\hline $\mathrm{Si}$ & $1.74_{-0.20}^{+0.20}$ & 0.08 & 1.2 & 0.23 & 18.6 & 0.18 \\
\hline S & $2.34_{-0.07}^{+0.04}$ & 0.26 & 17 & 0.30 & 22.4 & 0.17 \\
\hline $\mathrm{Ar}$ & $3.05_{-0.05}^{+0.06}$ & 0.062 & 3.4 & 0.08 & 9.2 & 0.066 \\
\hline $\mathrm{Ca}$ & $3.73_{-0.10}^{+0.08}$ & 0.018 & 0.06 & 0.067 & 1.3 & 0.051 \\
\hline $\mathrm{Fe}(\mathrm{K} \alpha)$ & $6.49_{-0.01}^{+0.01}$ & 3.1 & 520 & 2.9 & 540 & 3.0 \\
\hline $\mathrm{Fe}(\mathrm{K} \beta)$ & $7.18_{-0.05}^{+0.01}$ & 0.42 & 59 & 0.40 & 49 & 0.38 \\
\hline $\mathrm{Ni}$ & $7.56_{-0.14}^{+0.12}$ & 0.038 & 1.3 & 0.013 & 3.5 & 0.10 \\
\hline
\end{tabular}

aDerived fluorescent line intensities when the highly ionized line emission component is modeled as plasmas in CIE and PIE.

${ }^{\mathrm{b}}$ Measured line centroids in the PIE fit, which are fixed in the CIE fit.

${ }^{\mathrm{c}}$ In multiples of $10^{-4}$ photons $\mathrm{cm}^{-2} \mathrm{~s}^{-1}$

${ }^{\mathrm{d}}$ Line intensities expected from pure cold reflection normalized to the average observed Fe K $\alpha$ flux. 
Table 2. Summary of the Collisional Ionization Equilibrium Fit

\begin{tabular}{ll}
\hline \hline Parameter & \multicolumn{1}{c}{ Value } \\
\hline$N_{H}$ & $1.4 \times 10^{22} \mathrm{~cm}^{-2}$ \\
$\Gamma^{\mathrm{a}}$ & 1.5 \\
$L_{\text {soft }}{ }^{\mathrm{b}}$ & $3 \times 10^{40} \mathrm{erg} \mathrm{s}^{-1}$ \\
$k T$ & $0.69_{-0.07}^{+0.05} \mathrm{keV}$ \\
$E M$ & $\left(1.12_{-0.10}^{+0.17}\right) \times 10^{63} \mathrm{~cm}^{-3}$ \\
\hline
\end{tabular}

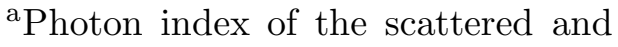
reflected continuum components, which are set equal in the fit.

${ }^{\mathrm{b}}$ Derived luminosity in the soft continuum component. 
Table 3. Continuum Parameters for the Photoionization Equilibrium Fit

\begin{tabular}{ccc}
\hline \hline & \multicolumn{2}{c}{ Values } \\
\cline { 2 - 3 } Parameter & Empirical & DEM \\
\hline$N_{H}\left(10^{22} \mathrm{~cm}^{-2}\right)$ & 0.87 & 0.89 \\
$\Gamma^{\mathrm{a}}$ & 1.8 & 1.6 \\
$L_{\mathrm{soft}}\left(\mathrm{erg} \mathrm{s}^{-1}\right)$ & $1.2 \times 10^{40}$ & $8.4 \times 10^{39}$ \\
\hline
\end{tabular}

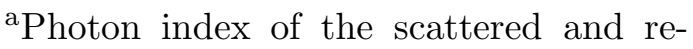
flected continuum components, which are set equal in the fit. 
Table 4. Summary of the Photoionization Ionization Equilibrium Fit

\begin{tabular}{ll}
\hline \hline Parameter & \multicolumn{1}{c}{ Value $^{\mathrm{a}}$} \\
\hline$\beta$ & $1.48_{-0.04}^{+0.03}$ \\
$\xi_{\min }$ & $0.91_{-\infty}^{+0.48}$ \\
$\xi_{\max }$ & $3.53_{-0.20}^{+0.32}$ \\
$E M$ & $5.5 \pm 0.7 \times 10^{63} \mathrm{~cm}^{-3}$ \\
\hline
\end{tabular}

${ }^{\text {a}}$ Errors correspond to $90 \%$ confidence ranges for two interesting parameters as described in the text. 